\title{
Worldline approach to quantum field theories on flat manifolds with boundaries
}

\section{Fiorenzo Bastianelli and Olindo Corradini}

Dipartimento di Física, Università di Bologna, and INFN, Sezione di Bologna

Via Irnerio, 46 - Bologna I-40126, Italy

E-mail: bastianelli@bo.infn.it, corradini@bo.infn.it

\section{Pablo A. G. Pisani}

IFLP, Departamento de Física, Facultad de Ciencias Exactas

UNLP, C.C. 67 (1900), La Plata, Argentina

E-mail: pisani@obelix.fisica.unlp.edu.ar

ABstract: We study a worldline approach to quantum field theories on flat manifolds with boundaries. We consider the concrete case of a scalar field propagating on $\mathbb{R}_{+} \times \mathbb{R}^{D-1}$ which leads us to study the associated heat kernel through a one dimensional (worldline) path integral. To calculate the latter we map it onto an auxiliary path integral on the full $\mathbb{R}^{D}$ using an image charge. The main technical difficulty lies in the fact that a smooth potential on $\mathbb{R}_{+} \times \mathbb{R}^{D-1}$ extends to a potential which generically fails to be smooth on $\mathbb{R}^{D}$. This implies that standard perturbative methods fail and must be improved. We propose a method to deal with this situation. As a result we recover the known heat kernel coefficients on a flat manifold with geodesic boundary, and compute two additional ones, $A_{3}$ and $A_{\frac{7}{2}}$. The calculation becomes sensibly harder as the perturbative order increases, and we are able to identify the complete $A_{\frac{I}{2}}$ with the help of a suitable toy model. Our findings show that the worldline approach is viable on manifolds with boundaries. Certainly, it would be desirable to improve our method of implementing the worldline approach to further simplify the perturbative calculations that arise in the presence of non-smooth potentials.

Keywords: Field Theories in Higher Dimensions, Field Theories in Lower Dimensions, Sigma Models. 


\section{Contents}

$\begin{array}{ll}\text { 1. Introduction } & 1\end{array}$

2. Heat kernel and path integrals 3

3. Heat kernel and path integrals on a flat space with boundary 6

3.1 The method 6

3.2 Particle on the half line 8

3.3 Toy model: $V(x)=a x \quad 14$

4. Conclusions

\section{Introduction}

The worldline approach to quantum field theory (QFT) has been extensively used to simplify calculations of many physical quantities like amplitudes, anomalies and effective actions, see [1] for a review. Recently this method has been extended to include the couplings of particles of spin $0,1 / 2$ and 1 to external gravity [2-4] together with some new applications [5]. Extensions to higher spin fields is also under study [6]. In this paper we investigate the possibility of using a worldline approach to quantum field theories defined on manifolds with boundaries. Previous investigations of the worldline path integral approach on manifolds with boundaries have already been carried out by using numerical Monte Carlo simulations and successfully applied to Casimir energy calculations $[7,8]$.

QFTs on manifolds with boundaries have always attracted a certain interest, with applications ranging from Casimir energies to critical phenomena near boundaries, including worldsheet approaches to open strings. More recently, they have drawn attention in the context of the Horava-Witten theory [9] and in the brane world scenarios, as for example in [10]. Thus, it seems useful to investigate and develop new calculational tools that may be used to study the properties of QFT with boundaries.

We investigate here an analytical approach based on first quantization, where a path integral over the coordinates of the field quanta is introduced. To reach directly the heart of the problem, we consider the case of a scalar field $\phi$ defined on a flat space with geodesic boundary, the $D$-dimensional manifold $\mathcal{M}=\mathbb{R}_{+} \times \mathbb{R}^{D-1}$. We use euclidean conventions and consider a generic potential $\mathrm{U}(\phi)$. The QFT action is given by

$$
S_{\mathrm{QFT}}[\phi]=\int_{\mathcal{M}} d^{D} x\left(\frac{1}{2} \partial_{\mu} \phi \partial^{\mu} \phi+\mathrm{U}(\phi)\right)
$$




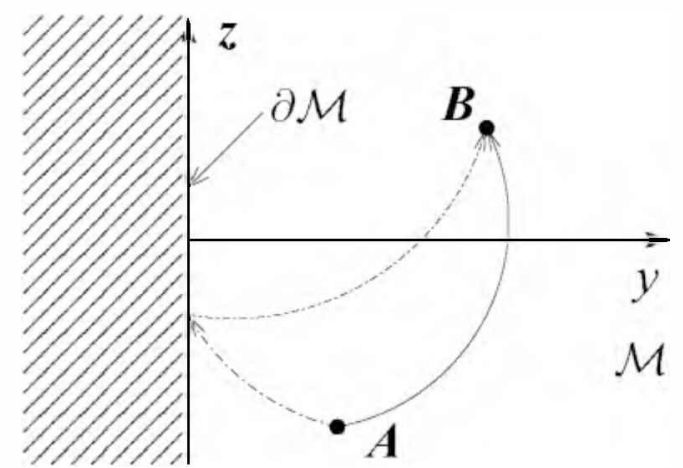

Figure 1: Quantum paths from the initial point $A$ to the final point $B$, in the flat space $\mathcal{M}$ with boundary $\partial \mathcal{M}$.

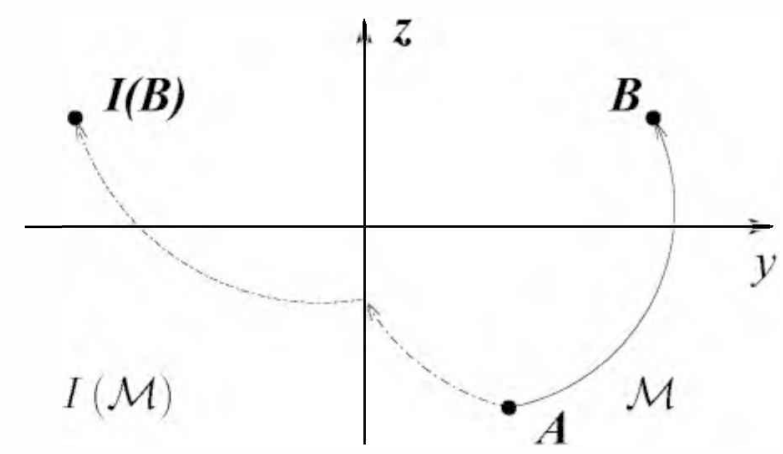

Figure 2: The image charge method: the bouncing path of figure 1 is mapped onto the path $A \rightarrow I(B)$.

and after specifying suitable boundary conditions (e.g. Dirichlet or Neumann) one may compute the corresponding one-loop effective action, which can be represented as

$$
\begin{aligned}
\Gamma[\phi] & =-\log \operatorname{Det}^{-\frac{1}{2}}\left[-\square+U^{\prime \prime}(\phi)\right]=-\frac{1}{2} \int_{0}^{\infty} \frac{d T}{T} \operatorname{Tr} e^{-T\left(-\square+U^{\prime \prime}(\phi)\right)} \\
& =-\frac{1}{2} \int_{0}^{\infty} \frac{d T}{T} \int_{P B C} \mathcal{D} x e^{-S|x|} .
\end{aligned}
$$

The path integral in the last line is evaluated with periodic boundary conditions (PBC) and contains the worldline action

$$
S[x]=\int_{0}^{T} d \tau\left(\frac{1}{4} \dot{x}^{\mu} \dot{x}_{\mu}+U^{\prime \prime}(\phi)\right)
$$

which produces the differential operator $-\square+U^{\prime \prime}(\phi)$ as quantum hamiltonian. The scalar field $\phi(x)$ is defined on $\mathcal{M}$ and, as a consequence, the paths $x^{\mu}(\tau)$ are restricted to lie on $\mathcal{M}$, see figure 1 . Thus, one has to develop tools to calculate worldline path integrals with such a restriction on the path integration variables.

General constructions of path integrals on target spaces with boundaries can be found in several textbooks, see for example [11, 12]. However, the time slicing constructions presented there do not seem to assist in practical perturbative calculations. One would like to have a reliable scheme to perform perturbative calculations in the presence of the boundary. For this purpose we find it convenient to introduce an image charge and map the original problem to a new one defined on the full space $\mathbb{R}^{I}$, see figure 2 . The major difficulty now arises from the fact that the potential extended on the full space may be nonsmooth at the fixed points of the $\mathbb{Z}_{2}$ identification that reintroduces the boundary. In this paper we develop a scheme to deal with this situation, and use it to compute perturbatively the matrix elements of the heat kernel $\left\langle y\left|e^{\left.-T \hat{i}-\square+U^{\prime \prime}(\phi)\right)}\right| x\right\rangle$ together with its trace, which appears in (1.2). The non-smooth part of the interaction potential on $\mathbb{R}^{D}$ is going to be due to the presence of certain step functions. Our scheme implements these step functions, which contain the quantum field and sit inside correlation functions, by transferring their effect to the limits of integration of suitably inserted completeness relations. 
Several methods for computing the trace of the heat kernel on manifolds with boundaries have been studied and used in the literature, as for example in [13-19]. For a review and additional references one may consult [20]. The method which is closer to the present one is presumably that developed by McAvity and Osborn [17], where the DeWitt ansatz [21] for the heat kernel is generalized to the case with boundaries. The novelty of our approach is that we use a path integral to calculate directly the heat kernel. An advantage of the path integral method is that it is quite intuitive and flexible, allowing to study the complete heat kernel and more general correlation functions, though we shall not investigate the latter here. Originally we also expected it to be quite an efficient method, but we find that this expectation is not fully realized, and presumably it can be improved by devising alternative and more efficient ways of calculating the perturbative expansion. Nevertheless, in this paper we are able to reach higher perturbative orders for the trace of the heat kernel than those we could find in the literature [18, 20], namely $A_{3}$ and $A_{\frac{7}{2}}$, see eqs. (3.27) and (3.28), but to identify the full $A_{z}$ we needed to use the result of a suitable toy model. In general, higher orders of the perturbative expansion produce multiple integrals that are difficult to compute explicitly, even though they could be computed numerically, perhaps using the methods of refs. $[7,8]$. We use the toy model to identify precisely the value of one of these integrals.

After reviewing in section 2 the path integral approach to the heat kernel on flat spaces without boundaries, we discuss in section 3 the case with a geodesic boundary, presenting first a brief description of our method and then its application to the half line and to $\mathbb{R}_{+} \times \mathbb{R}^{D-1}$. We give the heat kernel coefficients $A_{n}$ with $n$ integer and half integer up to $n=\frac{7}{2}$. We use a toy model to identify the value of a numerical coefficient entering the complete expression for $A_{\frac{7}{2}}$. Finally we present our conclusions and an outlook in section 4 .

\section{Heat kernel and path integrals}

Let us start reviewing the standard definition of the heat kernel and the way a path integral can be used to compute it. This will also serve to define our notations.

Given the differential operator corresponding to the hamiltonian of a nonrelativistic particle with unit mass

$$
H_{x}=-\frac{1}{2} \nabla_{x}^{2}+V(x)
$$

where $\nabla_{x}^{2}$ is the laplacian in the cartesian coordinates $x^{\mu}$ of flat space $\mathbb{R}^{D}$ and $V(x)$ an arbitrary potential, the heat kernel $K(x, y ; \beta)$ is the solution of the Schrödinger equation in euclidean time (the heat equation) with a particular boundary condition at time $\beta=0$

$$
\begin{aligned}
-\frac{\partial}{\partial \beta} K(x, y ; \beta) & =H_{x} K(x, y ; \beta) \\
K(x, y ; 0) & =\delta^{D}(x-y) .
\end{aligned}
$$

The heat kernel is formally given by the matrix element of the evolution operator $e^{-\beta H}$, which in the Dirac bracket notation reads

$$
K(x, y ; \beta)=\left\langle y\left|e^{-\beta H}\right| x\right\rangle
$$


and corresponds to the transition amplitude for the quantum system to start at point $x^{\mu}$ and reach point $y^{\mu}$ in an euclidean time $\beta$. After setting $\beta=i t$ it gives the transition amplitude corresponding to the Schrödinger equation in real minkowskian time $t$, but we prefer to use the Wick rotated version with euclidean time. ${ }^{1}$

Various methods for computing the heat kernel from the Schrödinger equation with arbitrary potentials $V(x)$ have been developed in the literature. One of the most useful is the path integral approach which produces $K(x, y ; \beta)$ as a sum over all paths $x^{\mu}(t)$ linking the initial point $x^{\mu}$ to the final point $y^{\mu}$ in a time $\beta$

$$
K(x, y ; \beta)=\int_{x(0)=x}^{x(\beta)=y} D x e^{-S \mid x]}
$$

where the euclidean action $S[x]$ in the exponent is the one leading to the hamiltonian $H_{x}$, i.e.

$$
S[x]=\int_{0}^{\beta} d t\left(\frac{1}{2} \delta_{\mu \nu} \dot{x}^{\mu} \dot{x}^{\nu}+V(x)\right) .
$$

The symbol $D x$ indicates the path integral measure

$$
D x=\prod_{0<t<\beta} d^{D} x(t)
$$

where the range of $x^{\mu}(t)$ is the full $\mathbb{R}^{D}$ for any $t$.

In general, it is not known how to compute the path integral for arbitrary potentials $V(x)$. A standard approximation method, useful in many contexts, is to compute the path integral in a perturbative expansion for small propagation time $\beta$ and small distances $\xi^{\mu} \equiv y^{\mu}-x^{\mu}$. It is often useful to introduce a rescaled time $\tau=t / \beta$ and write the action as

$$
S[x]=\frac{1}{\beta} \int_{0}^{1} d \tau\left(\frac{1}{2} \delta_{\mu \nu} \dot{x}^{\mu} \dot{x}^{\nu}+\beta^{2} V(x)\right)
$$

where the dots now refer to $\tau$ derivatives. Then one may split the action into a free part plus an interacting one

$$
\begin{aligned}
S[x] & =S_{0}[x]+S_{\mathrm{int}}[x] \\
S_{0}[x] & =\frac{1}{\beta} \int_{0}^{1} d \tau \frac{1}{\overline{2}} \delta_{\mu \nu} \dot{x}^{\mu} \dot{x}^{\nu}, \quad S_{\mathrm{int}}[x]=\frac{1}{\beta} \int_{0}^{1} d \tau \beta^{2} V(x) .
\end{aligned}
$$

The path integral for the free part $S_{0}[x]$ is exactly calculable, and the interaction part can be treated as a perturbation. The terms generated by the interaction potential $V(x)$, assumed to be smooth, can be computed by Wick contracting the quantum fields and evaluating the emerging Feynman diagrams. This method of computation gives an answer of the form

$$
K(x, y ; \beta)=\frac{1}{(2 \pi \beta)^{\frac{D}{2}}} e^{-S_{0}[\bar{x}]} \Omega(x, y ; \beta)
$$

\footnotetext{
${ }^{1}$ Substituting $\beta \rightarrow 2 T$ and $V(x) \rightarrow \frac{1}{2} \mathrm{U}(\phi(x))$ one finds the heat kernel needed for the scalar field $\phi$ described in the introduction.
} 
where $\bar{x}^{\mu}(\tau)=x^{\mu}+\left(y^{\mu}-x^{\mu}\right) \tau$ is the classical trajectory of the free equations of motion derived form $S_{0}[x]$, so that $S_{0}[\bar{x}]=\frac{1}{2 \cdot 3}(x-y)^{2}$. The term $(2 \pi \beta)^{-\frac{D}{2}}$ is the one loop correction which gives the correct normalization of the free path integral, and $\Omega(x, y ; \beta)$ contains the perturbative corrections due to a nonvanishing potential $V$. The perturbative calculation in terms of Feynman diagrams produces an answer of the form

$$
\Omega(x, y ; \beta) \sim \sum_{n=0}^{\infty} a_{n}(x, y) \beta^{n}
$$

where $a_{0}(x, y)=1$ in flat space. The coefficients $a_{n}(x, y)$ are often called Seeley-DeWitt coefficients and correspond to a $(n+1)$-loop calculation on the worldline. ${ }^{2}$ Thus we see that the DeWitt ansatz (2.9)-(2.10) for solving the heat equation emerges naturally from the path integral.

For example, assuming a smooth potential $V$, one may compute perturbatively to order $\beta^{\frac{7}{2}}$ (counting $\xi^{\mu}=y^{\mu}-x^{\prime \prime} \sim \sqrt{\beta}$ ) the transition amplitude

$$
\begin{aligned}
K(x, y ; \beta)=\frac{e^{-\xi^{2} \frac{2}{\beta}}}{(2 \pi \beta)^{\frac{D}{2}}} \exp [ & -\beta\left(1+\frac{1}{2} \xi \cdot \partial+\frac{1}{3 !}(\xi \cdot \partial)^{2}+\frac{1}{4 !}(\xi \cdot \partial)^{3}+\frac{1}{5 !}(\xi \cdot \partial)^{4}+\frac{1}{6 !}(\xi \cdot \partial)^{5}\right) V(x) \\
& -\frac{2 \beta^{2}}{4 !}\left(1+\frac{1}{2} \xi \cdot \partial+\frac{3}{20}(\xi \cdot \partial)^{2}+\frac{1}{30}(\xi \cdot \partial)^{3}\right) \square V(x) \\
& \left.+\frac{\beta^{3}}{4 !}\left(1+\frac{1}{2} \xi \cdot \partial\right)\left(\left(\partial_{\mu} V(x)\right)^{2}-\frac{1}{10} \square^{2} V(x)\right)+\cdots\right] .
\end{aligned}
$$

We have given the result in an exponentiated form since it is simpler to compute the connected worldline diagrams only. Note that in this compact notation one considers $[\xi, \partial]=0$. It is sometimes useful to present the result in a symmetrized form as follows

$$
\begin{aligned}
K(x, y ; \beta)= & \frac{e^{-\frac{\xi^{2}}{2 \beta}}}{(2 \pi \beta)^{\frac{D}{2}}} \exp \left[\beta\left(-\bar{V}+\frac{1}{12} \xi^{\mu} \xi^{\nu} \overline{\partial_{\mu} \partial_{\nu} V}-\frac{1}{5 !} \xi^{\mu} \xi^{\nu} \xi^{\alpha} \xi^{\beta} \overline{\partial_{\mu} \partial_{\nu} \partial_{\alpha} \partial_{\beta} V}\right)\right. \\
& \left.+\frac{\beta^{2}}{5 !}\left(-10 \overline{\square V}+\xi^{\mu} \xi^{\nu} \overline{\partial_{\mu} \partial_{\nu} \square V}\right)+\frac{\beta^{3}}{5 !}\left(5 \overline{\left(\partial_{\mu} V\right)^{2}}-\frac{1}{2} \overline{\square^{2} V}\right)+\cdots\right]
\end{aligned}
$$

where we have introduced the notation $\bar{V}=\frac{1}{2}(V(x)+V(y))$, etc., so that the result is written in a more compact form and the symmetry $x \leftrightarrow y$ is manifest.

Expanding the exponent one reads off the expansion in $\xi$ of the Seeley-DeWitt coefficients $a_{0}, a_{1}, a_{2}, a_{3}$

$$
\begin{aligned}
& a_{0}(x, y)=1 \\
& a_{1}(x, y)=-\bar{V}+\frac{1}{12} \xi^{\mu} \xi^{\nu} \overline{\partial_{\mu} \partial_{\nu} V}-\frac{1}{5 !} \xi^{\mu} \xi^{\nu} \xi^{\alpha} \xi^{\beta} \overline{\partial_{\mu} \partial_{\nu} \partial_{\alpha} \partial_{\beta} V}+O\left(\xi^{6}\right) \\
& a_{2}(x, y)=\frac{1}{2} \bar{V}^{2}-\frac{1}{5 !}\left(10 \overline{\square V}-\xi^{\mu} \xi^{\nu} \overline{\partial_{\mu} \partial_{\nu} \square V}\right)-\frac{1}{12} \xi^{\mu} \xi^{\nu} \bar{V} \overline{\partial_{\mu} \partial_{\nu} V}+O\left(\xi^{4}\right) \\
& a_{3}(x, y)=-\frac{1}{3 !} \bar{V}^{3}+\frac{1}{4 !}\left(\overline{\left(\partial_{\mu} V\right)^{2}}+2 \bar{V} \overline{\square V}-\frac{1}{10} \overline{\square^{2} V}\right)+O\left(\xi^{2}\right) .
\end{aligned}
$$

\footnotetext{
${ }^{2}$ This is generically true in curved space, as propagators go like 3 and vertices like $3^{-1}$. with 3 considered as the loop counting parameter. However in flat space the potential $V$ is the only source of vertices and contains an extra power of $k^{2}$ which offsets this counting: each vertex with $V$ increases the loop counting by 2 because of the factor $\beta^{2}$.
} 
Their values at coinciding points $y^{\mu}=x^{\mu}\left(\right.$ i.e. $\left.\xi^{\mu}=0\right)$ are easily obtained

$$
\begin{aligned}
& a_{0}(x, x)=1 \\
& a_{1}(x, x)=-V \\
& a_{2}(x, x)=\frac{1}{2} V^{2}-\frac{1}{12} \square V \\
& a_{3}(x, x)=-\frac{1}{3 !} V^{3}+\frac{1}{4 !}\left[\left(\partial_{\mu} V\right)^{2}+2 V \square V-\frac{1}{10} \square^{2} V\right] .
\end{aligned}
$$

As for higher orders, one may find in [22] the heat kernel coefficients $a_{n}(x, x)$ (modulo total derivatives) up to $n=8$ included, a result which has been obtained using a worldline approach.

\section{Heat kernel and path integrals on a flat space with boundary}

\subsection{The method}

Now let us consider the case of a space with a boundary. For definiteness we consider the flat space $\mathcal{M}=\mathbb{R}_{+} \times \mathbb{R}^{D-1}$ with coordinates $x^{\mu}=\left(y, z^{i}\right)$ where $0 \leq y<x$ and $z^{i} \in \mathbb{R}^{D-1}$. This space has a boundary $\partial \mathcal{M}=\mathbb{R}^{D-1}$ located at $y=0$, see figure 1 . We wish to obtain the heat kernel for the operator $H_{x}$ again as a path integral

$$
K\left(x_{1}, x_{2} ; \beta\right)=\int_{x(0)=x_{1}}^{x(1)=x_{2}} D x e^{-S[x]}
$$

with the same action $S[x]$ as in (2.7), but now summed over all paths $x^{\mu}(\tau)$ that lie in $\mathcal{M}$. In the presence of a boundary one must impose boundary conditions on $\partial \mathcal{M}$, and for simplicity we assume either Dirichlet boundary conditions, $K\left(x, x_{2} ; \beta\right)=0$ for $x \in \partial \mathcal{M}$, or Neumann boundary conditions, $\partial_{y} K\left(x, x_{2} ; \beta\right)=0$ for $x \in \partial \mathcal{M}$ where $\partial_{y}$ is the derivative normal to the boundary.

It is evident that on such a manifold the free action $S_{0}$ has two local minima corresponding to the straight classical path $\bar{x}_{1}(\tau)$ joining $x_{1}$ to $x_{2}$ and to the path $\bar{x}_{2}(\tau)$ which bounces once at the boundary, see figure 3 . The path integral can be computed by summing over all fluctuations around these two classical paths. This produces an answer of the form

$$
K\left(x_{1}, x_{2} ; \beta\right)=\frac{1}{(2 \pi \beta)^{\frac{D}{2}}}\left(e^{-S_{0}\left[\bar{x}_{1}\right]} \Omega_{1}\left(x_{1}, x_{2} ; \beta\right)+\gamma e^{-S_{0}\left|\bar{x}_{2}\right|} \Omega_{2}\left(x_{1}, x_{2} ; \beta\right)\right)
$$

where the $\Omega_{i}\left(x_{1}, x_{2} ; \beta\right)$ contain the perturbative loop corrections around the classical paths $\bar{x}_{\imath}(\tau)$, and $\gamma$ is a relative phase between these paths which depends on the boundary conditions chosen on $\partial \mathcal{M}$. This formula reproduces the generalized DeWitt ansatz introduced by McAvity and Osborn to study the heat kernel on spaces with boundaries [17]. In the following we describe a method for computing the path integral and obtain perturbatively the functions $\Omega_{2}\left(x_{1}, x_{2} ; \beta\right)$. In particular we will focus on the calculations of the trace of the heat kernel

$$
\operatorname{Tr} e^{-\beta H}=\int_{\mathcal{M}} d^{D} x K(x, x ; \beta)=\int_{P B C} D x e^{-S[x]}
$$




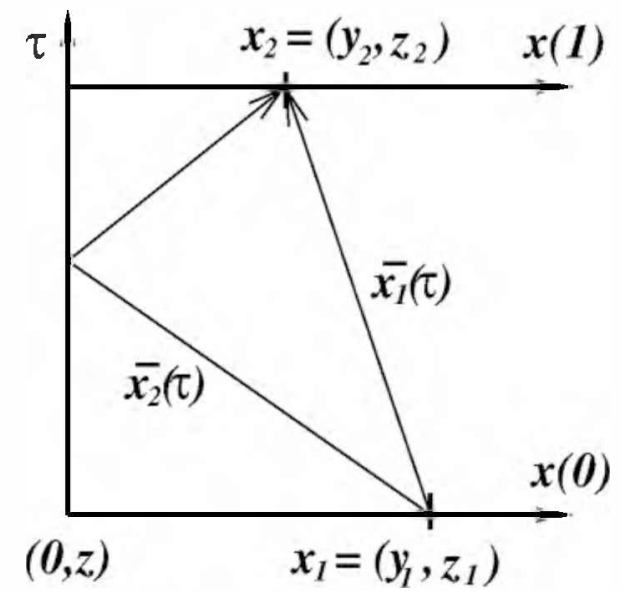

Figure 3: Classical paths in the flat space with boundary $\mathcal{M}$. On the vertical axis we have placed the time $\tau$.

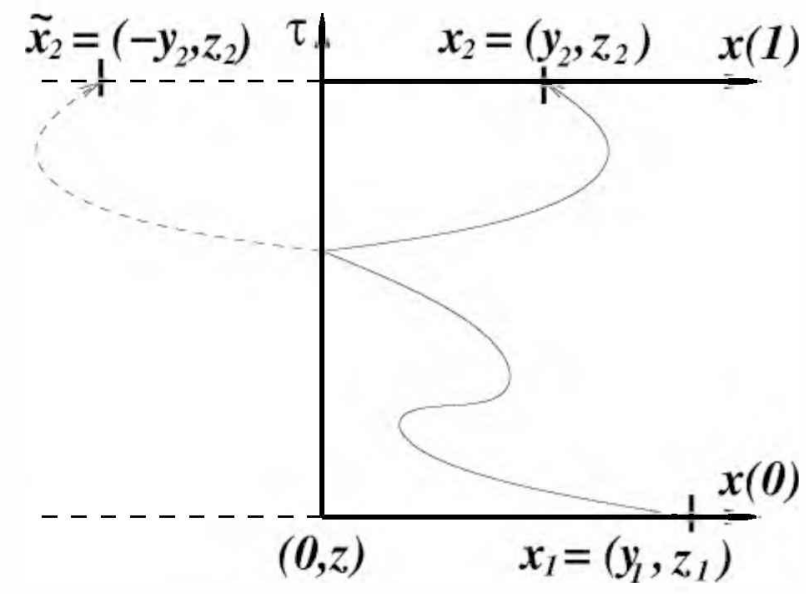

Figure 4: A bouncing quantum path and its image on $\mathbb{R}^{D}$.

where $P B C$ denote periodic boundary conditions for the trajectories on $\mathcal{M}$.

For evaluating the path integral over $\mathcal{M}$ it is convenient to use the method of images and double the space to obtain the full $\mathbb{R}^{L}$. Trajectories that bounce at the boundary are traded for trajectories that continue off the boundary with a reflected path, see figure 4 . A trajectory extended on the full $\mathbb{R}^{D}$ (dashed line in figure 4) contributes the same weight in the path integral as the original one (solid line), provided the potential is extended on $\mathbb{R}^{D}$ as an even function under the reflection $y \rightarrow-y$

$$
V(x) \rightarrow \dot{V}(x)=\theta(y) V(y, z)+\theta(-y) V(-y, z)
$$

where $\theta$ is the Heaviside (step) function.

Dirichlet or Neumann boundary conditions are special cases of the generic ansatz (3.2): they correspond to setting $\gamma=-1$ or $\gamma=+1$, respectively. Hence, the transition amplitude for such cases may be expressed as

$$
K_{\mathcal{M}}\left(x_{1}, x_{2} ; \beta\right)=K_{\mathbb{R}^{D}}\left(x_{1}, x_{2} ; \beta\right) \mp K_{\mathbb{R}^{D}}\left(x_{1}, \tilde{x}_{2} ; \beta\right) .
$$

The heuristic interpretation for $\gamma=-1$ is quite clear. In fact, notice that the second term in (3.5) involves paths that cross the boundary at least once. Hence, it is easy to convince oneself that - due to the symmetry of the potential - the only effect of such a term is to cancel all those paths in the first term that hit the boundary. In fact, setting $y_{2}=0$ (all paths now hit the boundary) leads to a vanishing kernel: therefore the wave function evolved with the kernel

$$
\psi\left(x_{2}, \beta\right)=\int_{\mathcal{M}} d x_{1} K_{\mathcal{M}}\left(x_{1}, x_{2} ; \beta\right) \psi\left(x_{1}, 0\right)
$$

satisfies Dirichlet boundary conditions. For $\gamma=+1$ we know of no simple heuristic interpretation; however it is clear that differentiating (3.5) with respect to $y_{2}$ and setting 
$y_{2}=0$ leads to a vanishing result: the normal derivative of the wave function vanishes at the boundary. Hence, $\gamma=+1$ corresponds to Neumann boundary conditions.

The problem has thus been reduced to a path integral computation on a boundaryless manifold. However the path integral on $\mathbb{R}^{D}$ with the potential $\vec{V}$ is not easily computable by Taylor expanding the potential and using Wick contractions because of the step function $\theta(y)$ contained in $\bar{V}$. Even assuming that $V(x)$ is smooth enough to admit a Taylor expansion, $\bar{V}(x)$ in general is not.

Nevertheless, as a test, one could try to insert $\tilde{V}$ into (2.11), which was obtained by assuming smoothness of the potential. Evaluating the derivatives contained in (2.11) on $\bar{V}$ would then produce delta functions and derivatives thereof, which use seems rather unwarranted and difficult to implement consistently.

Thus one has to study methods to compute perturbatively path integrals that contain step functions of the quantum fields. A method to address this problem is the following one. Whenever a step function containing the quantum field $q(\tau)$ appears inside correlation function, such as $\left\langle\theta\left(q\left(\tau_{1}\right)-a\right)\right\rangle$, one may split the path integral into two parts using a completeness relation at time $\tau_{1}$ (namely $\mathbb{I}=\int_{-\infty}^{\infty} d q_{1}\left|q_{1}\right\rangle\left\langle q_{1}\right|$ ) to transfer the constraint required by the step function into the limit of integration - namely $\int_{a}^{\infty} d q_{1}$. Each of the two remaining parts are standard path integrals without step functions, but have boundary conditions linked by the $\int_{a}^{\infty} d q_{1}$ integration.

Next we will exemplify this method to compute the heat kernel expansion for a particle on the half line. This $D=1$ case is enough for our purposes, since it contains the essential information. At the end of the section we shall reconstruct the complete results for generic D.

\subsection{Particle on the half line}

We consider here the heat kernel on the half line $\mathbb{R}_{+}$and denote by $x$ its single coordinate. For Dirichlet/Neumann boundary conditions the expression for the transition amplitude reads (in an obvious notation)

$$
\left\langle x_{2}\left|e^{-\beta H}\right| x_{1}\right\rangle_{\mathbb{R}_{+}}=\left\langle x_{2}, \beta \mid x_{1}, 0\right\rangle_{\mathbb{R}_{+}}=\left\langle x_{2}, \beta \mid x_{1}, 0\right\rangle_{\mathbb{R}} \mp\left\langle\tilde{x}_{2}, \beta \mid x_{1}, 0\right\rangle_{\mathbb{R}}
$$

where $\bar{x}_{2}=-x_{2}$ is the coordinate of the image charge. We now proceed to compute the path integral for the two terms on the right hand side, as explained earlier.

As usual in path integral computations one splits the quantum paths into a classical path $x_{c l}(\tau)$, satisfying the equations of motion, and quantum fluctuations $q(\tau)$. We associate the boundary conditions with the classical path so that the quantum fluctuations $q(\tau)$ have vanishing boundary conditions

$$
\begin{aligned}
& x(\tau)=x_{c l}(\tau)+q(\tau)=x_{1}+\left(x_{2}-x_{1}\right) \tau+q(\tau), \\
& q(0)=q(1)=0 .
\end{aligned}
$$

We treat the potential $\vec{V}$ as a perturbation for the free particle. Hence, the path integral 


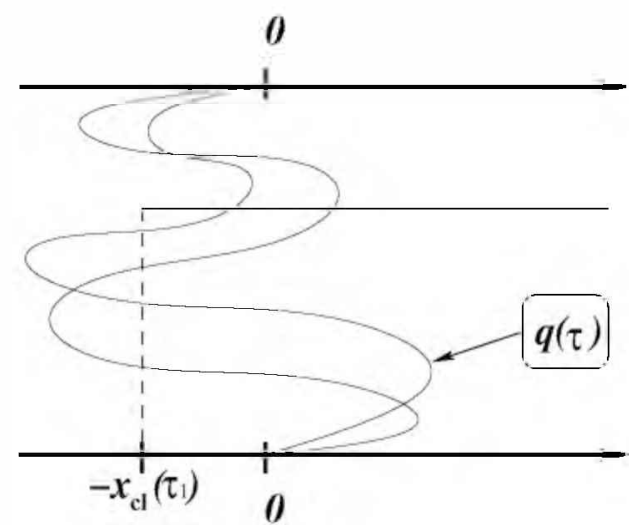

Figure 5: Quantum paths constrained by $\theta\left(x_{c l}\left(\tau_{1}\right)+q\left(\tau_{1}\right)\right)$. The time $\tau$ runs along the vertical axis.

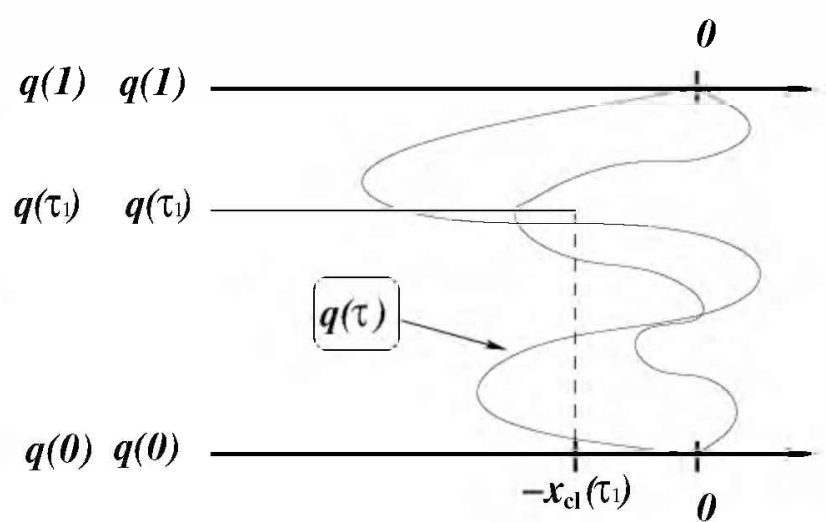

Figure 6: Quantum paths constrained by $\theta\left(-x_{c l}\left(\tau_{1}\right)-q\left(\tau_{1}\right)\right)$.

for the full line, appearing in (3.7), is given by

$$
\begin{aligned}
\left\langle x_{2}, \beta \mid x_{1}, 0\right\rangle_{\mathbb{R}}= & e^{-S_{0}\left[x_{c l}\right]} \int_{q(0)=0}^{q(1)=0} D q e^{-S_{0}[q]} \exp \left(-\beta \int_{0}^{1} d \tau \tilde{V}\left(x_{c l}(\tau)+q(\tau)\right)\right) \\
= & e^{-\frac{1}{2 y}\left(x_{3}-x_{1}\right)^{2}} \int_{q(0)=0}^{q(1)=0} D q e^{-S_{0}[q]}\left(1-\beta \int_{0}^{1} d \tau_{1} \tilde{V}\left(x_{c l}\left(\tau_{1}\right)+q\left(\tau_{1}\right)\right)\right. \\
& \left.+\frac{\beta^{2}}{2 !} \int_{0}^{1} d \tau_{1} \int_{0}^{1} d \tau_{2} \bar{V}\left(x_{c l}\left(\tau_{1}\right)+q\left(\tau_{1}\right)\right) \hat{V}\left(x_{c l}\left(\tau_{2}\right)+q\left(\tau_{2}\right)\right)+O\left(\bar{V}^{3}\right)\right)
\end{aligned}
$$

where $S_{0}$ is the action for the free particle, $\vec{V}=0$. The first term in the expansion simply yields the path integral normalization

$$
\int_{q(0)=0}^{q(1)=0} D q e^{-S_{0}[q]}=\frac{1}{(2 \pi \beta)^{\frac{1}{2}}}
$$

whereas the rest contributes to the perturbative corrections to the heat kernel.

We have managed to compute the short-time perturbative expansion of the transition amplitude up to order $\beta^{\frac{7}{2}}$, considering $x_{1}-x_{2} \sim \sqrt{\beta}$. In the following we only give a detailed description of the contributions coming from a single insertion of the perturbation $V$, since this is enough to exemplify the method discussed previously. In principle this method can be applied to all orders in $V$.

To compute the aforementioned contributions we first extract the integral over the worldline (namely $\int_{0}^{1} d \tau_{1}$ ) out of the path integral; we will perform it at the end. Then, using (3.4), we have

$$
\begin{aligned}
\int_{q(0)=0}^{q(1)=0} D q e^{-S_{1} \mid q}[ & \theta\left(x_{c l}\left(\tau_{1}\right)+q\left(\tau_{1}\right)\right) V\left(x_{c l}\left(\tau_{1}\right)+q\left(\tau_{1}\right)\right) \\
& \left.+\theta\left(-x_{c l}\left(\tau_{1}\right)-q\left(\tau_{1}\right)\right) V\left(-x_{c l}\left(\tau_{1}\right)-q\left(\tau_{1}\right)\right)\right] .
\end{aligned}
$$


Note that the constraints for the quantum field $q(\tau)$, associated with the Heaviside functions, are localized at time $\tau=\tau_{1}$. Each of the two terms in (3.11) can thus be manipulated by splitting the path integral into two parts, as follows. In the first term the constraint acts as depicted in figure 5: we can thus compute the path integral as a convolution between a free path integral with boundary conditions $q(0)=0, q\left(\tau_{1}\right)=y$ and a free path integral with boundary conditions $q\left(\tau_{1}\right)=y, q(1)=0$, where $y$ is integrated over and satisfies the constraint $y \geq-x_{c l}\left(\tau_{1}\right)$ because of the step function. We thus have

$$
\begin{aligned}
\int_{q(0)=0}^{q(1)=0} D q e^{-S_{0}[q]} & \theta\left(x_{c l}\left(\tau_{1}\right)+q\left(\tau_{1}\right)\right) V\left(x_{c l}\left(\tau_{1}\right)+q\left(\tau_{1}\right)\right) \\
& =\int_{-x_{c l}\left(\tau_{1}\right)}^{\infty} d y\left(\int_{q(0)=0}^{q\left(\tau_{1}\right)=y} D q e^{\left.-S_{0} \mid q\right\}}\right) V\left(x_{c l}\left(\tau_{1}\right)+y\right)\left(\int_{q\left(\tau_{1}\right)=y}^{q(1)=0} D q e^{-S_{0}[q]}\right) \\
& =\int_{-x_{c l}\left(\tau_{1}\right)}^{\infty} d y \frac{e^{-\frac{1}{2 \beta \tau_{1}\left(1-\tau_{1}\right)} y^{2}}}{2 \pi \beta \sqrt{\tau_{1}\left(1-\tau_{1}\right)}} V\left(x_{c l}\left(\tau_{1}\right)+y\right)
\end{aligned}
$$

In a similar fashion, in the second term of (3.11) the constraint acts as depicted in figure 6 , and the path integral reads

$$
\begin{array}{r}
\int_{\substack{q(0)=0 \\
q(1)=0} q} e^{-S_{0}[q]} \theta\left(-x_{c l}\left(\tau_{1}\right)-q\left(\tau_{1}\right)\right) V\left(-x_{c l}\left(\tau_{1}\right)-q\left(\tau_{1}\right)\right) \\
=\int_{-\infty}^{-x_{c l}\left(\tau_{1}\right)} d y \frac{e^{-\frac{1}{2 \beta \tau_{1}\left(1-\tau_{1}\right)} y^{2}}}{2 \pi \beta \sqrt{\tau_{1}\left(1-\tau_{1}\right)}} V\left(-x_{c l}\left(\tau_{1}\right)-y\right) .
\end{array}
$$

Hence, the complete contribution of a single $V$ insertion to the whole line path integral can be written as

$$
\begin{aligned}
& \beta \frac{e^{-\frac{1}{2 \beta}\left(x_{2}-x_{1}\right)^{2}}}{(2 \pi \beta)^{\frac{1}{2}}} \int_{0}^{1} d \tau \frac{1}{\sqrt{2 \pi \beta \tau(1-\tau)}}\left(-\int_{-\infty}^{+\infty} d y e^{-\frac{y^{2}}{2 \beta \tau(1-\tau)}} V\left(x_{c l}(\tau)+y\right)\right. \\
& \left.+\int_{-\infty}^{-x_{c l}(\tau)} d y e^{-\frac{y^{2}}{2 \beta \tau(1-\tau)}}\left[V\left(x_{c l}(\tau)+y\right)-V\left(-x_{c l}(\tau)-y\right)\right]\right)
\end{aligned}
$$

where the constraint only sits in the integration limit of the second term. Higher order contributions in $V$ can analogously be split into one unconstrained part where the "quantum field" $y=q(\tau)$ may run over the whole real axis, and parts that depend upon the constraints: the latter are parity-odd. A few observations are in order.

- For an even potential $V$ the parity-odd terms vanish identically. In such a case it is possible to write the heat kernel as a power series in integer powers of $\beta$ and $x_{2}-x_{1}$. In fact, upon Taylor expanding the potential about the initial point $x_{1}$, the unconstrained gaussian integral over $y$ singles out only integer powers of $\beta$, and one reproduces the expression (2.12), with $x=x_{1}, y=x_{2}$ and $\xi=x_{2}-x_{1}$, plus an image charge contribution. We can thus write down the final expression for the heat kernel 
on the half line

$$
\begin{aligned}
\left\langle x_{2}, \beta \mid x_{1}, 0\right\rangle_{\mathbb{R}_{+}}= & \frac{e^{-\frac{\xi^{2}}{2 \beta}}}{(2 \pi \beta)^{\frac{1}{2}}} \exp \left[-\beta \bar{V}-\frac{\beta^{2}}{2 \cdot 3 !} \overline{\partial^{2} V}\left(1-\xi^{2} / \beta\right)\right. \\
& \left.-\frac{\beta^{3}}{2 \cdot 5 !} \overline{\partial^{4} V}\left(1-2 \xi^{2} / \beta+2 \xi^{4} / \beta^{2}\right)+\frac{\beta^{3}}{4 !} \overline{\partial V}^{2}+O\left(\beta^{4}\right)\right] \\
& \mp \frac{e^{-\frac{\xi^{2}}{2 \sigma}}}{(2 \pi \beta)^{\frac{1}{2}}} \exp \left[-\beta \bar{V}-\frac{\beta^{2}}{2 \cdot 3 !} \overline{\partial^{2} V}\left(1-\tilde{\xi}^{2} / \beta\right)\right. \\
& \left.-\frac{\beta^{6}}{2 \cdot 5 !} \overline{\partial^{4} V}\left(1-2 \bar{\xi}^{2} / \beta+2 \tilde{\xi}^{4} / \beta^{2}\right)+\frac{\beta^{3}}{4 !} \overline{\partial V}^{2}+O\left(\beta^{4}\right)\right]
\end{aligned}
$$

where $\bar{\xi}=-x_{2}-x_{1}$. The expansion above clearly corresponds to the McAvity-Osborn ansatz (3.2), with $\Omega_{i}$ 's both expressed as integer power series in $\beta$. In the present case with even potentials, it is clear that the heat kernel is also correctly reproduced by the conventional perturbative calculation of the path integral through the Wick's theorem, as discussed in section 2 .

- For a generic potential $V$, the constraint-independent part of the expansion yields again the expression (3.15), except that now $\bar{V}=\frac{1}{2}\left(V\left(-x_{2}\right)+V\left(x_{1}\right)\right)$ must appear in the second exponential in place of $\bar{V}=\frac{1}{2}\left(V\left(x_{2}\right)+V\left(x_{1}\right)\right)$, and so on. However, the constraint-dependent part now is not vanishing and does not seem to be naively expressible as a power series in $\beta$ and $x_{2}-x_{1}$, as the latter appears nontrivially in the integration limits. This complication corresponds to the difficulty of giving a reliable interpretation of the Taylor expansion of the Heaviside function, pointed out in the previous section.

- The method described above allows to compute the perturbative expansion in $\beta$ of the partition function

$$
\begin{aligned}
\operatorname{Tr} e^{-\beta \hat{H}} & =\int_{0}^{\infty} d x\langle x, \beta \mid x, 0\rangle_{\mathbb{R}_{+}} \\
& =\int_{0}^{\infty} d x\langle x, \beta \mid x, 0\rangle_{\mathbb{R}} \mp \int_{0}^{\infty} d x\langle-x, \beta \mid x, 0\rangle_{\mathbb{R}}
\end{aligned}
$$

for which we need to specialize expression (3.14), and analogous higher-order contributions, to:

(i) $x_{1}=x_{2}=x \quad \Longrightarrow \quad x_{c l}(\tau)=x$

and

(ii) $x_{1}=-x_{2}=x \quad \Longrightarrow \quad x_{c l}(\tau)=x(1-2 \tau)$.

Thus we can proceed to compute the small time expansion of the partition function up to order $\beta^{\frac{7}{2}}$ : 
(i) In this case, the constraint-independent parts of the potential insertions, such as the first term of (3.14), yield bulk contributions, whereas the constraint-dependent parts, after Taylor expanding the potential about the boundary, single out boundary terms. Hence, after some tedious algebra, we have

$$
\begin{aligned}
\int_{0}^{\infty} d x\langle x, \beta \mid x, 0\rangle_{\mathbb{R}}= & \frac{1}{(2 \pi \beta)^{\frac{1}{2}}}\left[\int _ { 0 } ^ { \infty } d x \operatorname { e x p } \left(-\beta V(x)-\beta^{2} \frac{\partial^{2} V(x)}{2 \cdot 3 !}+\beta^{3} \frac{(\partial V(x))^{2}}{4 !}\right.\right. \\
& \left.-\beta^{3} \frac{\partial^{4} V(x)}{2 \cdot 5 !}\right)-\beta^{2} \frac{\partial V(0)}{2 \cdot 3 !}+\beta^{3}\left(\frac{V \partial V(0)}{2 \cdot 3 !}-\frac{\partial^{3} V(0)}{2 \cdot 5 !}\right) \\
& \left.+\sqrt{\frac{\pi}{8}} \beta^{\frac{\pi}{2}} \frac{(\partial V(0))^{2}}{2^{6}}+O\left(\beta^{4}\right)\right]
\end{aligned}
$$

(ii) For such a case, the presence of the non trivial classical action $S_{0}\left[x_{c l}\right]=2 x^{2} / \beta$ allows for a Taylor expansion of the potential about the boundary location: the integral over the manifolds is convergent and gives

$$
\begin{aligned}
\int_{0}^{\infty} d x\langle-x, \beta \mid x, 0\rangle_{\mathbb{R}}= & \frac{1}{(2 \pi \beta)^{\frac{1}{2}}}\left\{\sqrt { \frac { \pi \beta } { 8 } } \left[1-\beta V(0)+\frac{\beta^{2}}{2}\left(V^{2}(0)-\frac{\partial^{2} V(0)}{4}\right)\right.\right. \\
& \left.+\beta^{3}\left(-\frac{V^{3}(0)}{3 !}+\frac{3(\partial V(0))^{2}}{2^{4}}-\frac{\partial^{4} V(0)}{2^{7}}\right)\right]-\beta^{2} \frac{\partial V(0)}{4} \\
& \left.+\beta^{3}\left(\frac{V \partial V(0)}{4}-\frac{\partial^{3} V(0)}{2 \cdot 4 !}\right)+O\left(\beta^{4}\right)\right\}
\end{aligned}
$$

Combining (3.17) and (3.18) we get the final result

$$
\begin{aligned}
& \operatorname{Tr} e^{-\beta \hat{H}}=\int_{0}^{\infty} d x\langle x, \beta \mid x, 0\rangle_{\mathbb{R}_{+}} \\
& =\frac{1}{(2 \pi \beta)^{\frac{1}{2}}}\left[\int _ { 0 } ^ { \infty } d x \operatorname { e x p } \left(-\beta V(x)-\beta^{2} \frac{\partial^{2} V(x)}{2 \cdot 3 !}+\beta^{3} \frac{(\partial V(x))^{2}}{4 !}\right.\right. \\
& \left.-\beta^{3} \frac{\partial^{4} V(x)}{2 \cdot 5 !}\right)+\sqrt{\frac{\pi}{8}} \beta^{\frac{7}{2}} \frac{(\partial V(0))^{2}}{2^{6}} \\
& \mp \sqrt{\frac{\pi \beta}{8}}\left[1-\beta V(0)+\frac{\beta^{2}}{2}\left(V^{2}(0)-\frac{\partial^{2} V(0)}{4}\right)\right. \\
& \left.+\beta^{3}\left(-\frac{V^{3}(0)}{3 !}+\frac{V \partial^{2} V(0)}{2^{3}}+\frac{(\partial V(0))^{2}}{4 !}-\frac{\partial^{4} V(0)}{2^{7}}\right)\right] \\
& \left.+\beta^{2}\left\{\begin{array}{c}
1 \\
-2
\end{array}\right\} \frac{\partial V(0)}{3 !}+\beta^{3}\left(\left\{\begin{array}{c}
-1 \\
2
\end{array}\right\} \frac{V \partial V(0)}{3 !}+\left\{\begin{array}{c}
2 \\
-3
\end{array}\right\} \frac{\partial^{3} V(0)}{5 !}\right)+O\left(\beta^{4}\right)\right] .
\end{aligned}
$$


This is the expansion of the partition function with Dirichlet/Neumann boundary conditions, valid up to order $\beta^{\frac{\tau}{2}}$, where the upper coefficient in the symbol $\{:\}$ refers to Dirichlet boundary conditions, and the lower one to Neumann boundary conditions. At this point the integrated heat kernel coefficients for the half line can be read off immediately. However, it is not difficult to reconstruct such coefficients for arbitrary $D$. Defining

$$
\operatorname{Tr} e^{-\beta H} \sim \frac{1}{(2 \pi \beta)^{\frac{D}{2}}} \sum_{n \in \mathbb{N} / 2} A_{n} \beta^{n} .
$$

we obtain

$$
\begin{aligned}
A_{0}= & \int_{\mathcal{M}} 1 \\
A_{\frac{1}{2}}= & \mp \sqrt{\frac{\pi}{8}} \int_{\partial \mathcal{M}} 1 \\
A_{1}= & -\int_{\mathcal{M}} V \\
A_{\frac{3}{2}}= & \pm \sqrt{\frac{\pi}{8}} \int_{\partial \mathcal{M}} V \\
A_{2}= & \int_{\mathcal{M}}\left(\frac{V^{2}}{2}-\frac{\square V}{12}\right)+\left\{\begin{array}{c}
1 \\
-2
\end{array}\right\} \int_{\partial \mathcal{M}} \frac{\partial_{y} V}{3 !} \\
A_{\frac{5}{2}}= & \mp \sqrt{\frac{\pi}{8}} \int_{\partial \mathcal{M}}\left[\frac{V^{2}}{2}-\left(\frac{\partial_{y}^{2}}{8}+\frac{\partial_{i}^{2}}{12}\right) V\right] \\
A_{3}= & \int_{\mathcal{M}}\left(-\frac{V^{3}}{3 !}+\frac{\left(\partial_{\mu} V\right)^{2}}{4 !}+\frac{V \square V}{2 \cdot 3 !}-\frac{\square^{2} V}{2 \cdot 5 !}\right) \\
& +\int_{\partial \mathcal{M}}\left[\{-1\} \frac{V \partial_{y} V}{3 !}+\left\{\begin{array}{l}
2 \\
2
\end{array}\right\} \frac{\partial_{y}^{3} V}{5 !}+\left\{\begin{array}{l}
3 \\
-3
\end{array}\right\} \frac{\partial_{y}^{4} \partial_{i}^{2} V}{2 \cdot 5 !}\right] \\
A_{\frac{7}{2}}= & \sqrt{\frac{\pi}{8}} \int_{\partial \mathcal{M}}\left[\mp\left[-\frac{\partial_{y}^{2} \partial_{i}^{2}}{4 \cdot 4 !}+\frac{\partial_{i}^{4}}{10 \cdot 4 !}\right) V \mid+\left\{\begin{array}{l}
7 \\
3 !
\end{array}\right]\right.
\end{aligned}
$$

Before concluding this subsection let us mention some caveats of our method. We have found it increasingly harder to calculate explicitly the numerical value of some terms. On the other hand our method allows to write all such numerical values in terms of multiple integrals of finite functions over compact domains which could eventually be calculated numerically. Since in the present manuscript we only encountered difficulties for one numerical coefficient in $A_{\frac{7}{2}}$, and precisely the one related to $\left(\partial_{y} V\right)^{2}$, we decided to fix it by using a toy model, which is discussed next. 


\subsection{Toy model: $V(x)=a x$}

The asymptotic expansion of $e^{-i 3 H}$ for small $\beta$ can also be obtained from the large- $\lambda$ asymptotic expansion of the resolvent $(H+\lambda)^{-1}$. Indeed, since the trace of the resolvent is the Laplace transform of the heat kernel trace, it admits the following representation for large $\lambda[20]$

$$
\operatorname{Tr}(H+\lambda)^{-1} \sim \frac{1}{\sqrt{2 \pi}} \sum_{n \in \mathbb{N} / 2} \Gamma\left(n+\frac{1}{2}\right) A_{n} \lambda^{-n-\frac{1}{2}}
$$

We use such an expansion to compute the $\left(\partial_{y} V\right)^{2}$ term in the coefficient $A_{\frac{7}{2}}$, considering a toy model that can be explicitly solved in the half line, namely the one-dimensional Schrödinger operator $H=-\frac{1}{2} \partial_{x}^{2}+a x$ with $x \in \mathbb{R}^{+}$and $a$ a real constant. We can compute the contribution to $A_{\frac{7}{3}}$ proportional to $\left(\partial_{y} V\right)^{2}$ from the order $\lambda^{-4}$ term of the asymptotic expansion, since the complete $A_{\frac{7}{2}}$ for the linear potential reduces to

$$
A_{\frac{7}{2}}=k \sqrt{\frac{\pi}{8}} \frac{\left(\partial_{y} V\right)^{2}}{2^{6}}
$$

with $\left(\partial_{y} V\right)^{2}=a^{2}$ for a constant $k$ which has to be fixed.

From the eigenfunctions of $H$ one obtains a simple expression for the kernel $G\left(x, x^{\prime}, \lambda\right)$ of the resolvent $(H+\lambda)^{-1}$

$$
G\left(x, x^{\prime}, \lambda\right)=-\frac{2}{W}\left\{\theta\left(x^{\prime}-x\right) L(x) R\left(x^{\prime}\right)+\theta\left(x-x^{\prime}\right) L\left(x^{\prime}\right) R(x)\right\}
$$

where $R(x)$ can be written in terms of the modified Bessel function $K_{\nu}(z)$

$$
R(x)=\sqrt{a x+\lambda} K_{1 / 3}\left(\frac{[2(a x+\lambda)]^{3 / 2}}{3 a}\right)
$$

and $L(x)$ is given by

$$
\begin{aligned}
L(x)= & \sqrt{a x+\lambda}\left\{K_{1 / 3}\left(\frac{(2 \lambda)^{3 / 2}}{3 a}\right) I_{1 / 3}\left(\frac{|2(a x+\lambda)|^{3 / 2}}{3 a}\right)-\right. \\
& \left.\left.-I_{1 / 3}\left(\frac{(2 \lambda)^{3 / 2}}{3 a}\right) K_{1 / 3}\left(\frac{|2(a x+\lambda)|^{3 / 2}}{3 a}\right)\right]\right\}
\end{aligned}
$$

for Dirichlet boundary conditions at $x=0$, and

$$
\begin{aligned}
L(x)= & \sqrt{a x+\lambda}\left\{\sqrt { 2 } \lambda \left[K_{1 / 3}^{\prime}\left(\frac{(2 \lambda)^{3 / 2}}{3 a}\right) I_{1 / 3}\left(\frac{\left[2(a x+\lambda i]^{3 / 2}\right.}{3 a}\right)-\right.\right. \\
& \left.-I_{1 / 3}^{\prime}\left(\frac{(2 \lambda)^{3 / 2}}{3 a}\right) K_{1 / 3}\left(\frac{[2(a x+\lambda)]^{3 / 2}}{3 a}\right)\right]+ \\
+ & \frac{a}{2 \sqrt{\lambda}}\left[K_{1 / 3}\left(\frac{(2 \lambda)^{3 / 2}}{3 a}\right) I_{1 / 3}\left(\frac{[2(a x+\lambda)]^{3 / 2}}{3 a}\right)-\right. \\
& \left.\left.-I_{1 / 3}\left(\frac{(2 \lambda)^{3 / 2}}{3 a}\right) K_{1 / 3}\left(\frac{[2(a x+\lambda)]^{3 / 2}}{3 a}\right)\right]\right\}
\end{aligned}
$$


for Neumann boundary conditions at $x=0 . W$ is the Wronskian between the corresponding $L(x)$ and $R(x)$.

From these expressions one can obtain the bulk and boundary contributions to the asymptotic expansion of the resolvent trace

$$
\operatorname{Tr}(H+\lambda)^{-1}=\int_{0}^{\infty} d x G(x, x, \lambda) .
$$

From the asymptotic behavior of the modified Bessel functions for large values of their arguments, it is simple to see that the boundary contributions of order $\lambda^{-4}$ and proportional to $a^{2}$ are given by $-\frac{15}{128} a^{2} \lambda^{-4}$ for Dirichlet boundary conditions, and $\frac{21}{128} a^{2} \lambda^{-4}$ for Neumann boundary conditions. Comparing these values with (3.29) and (3.30), we obtain $k=-5$ for Dirichlet boundary conditions, and $k=7$ for Neumann boundary conditions. These are the explicit numerical values of some multiple integrals, produced by our general method, that we were not able to evaluate directly.

\section{Conclusions}

We have developed a path integral method to generalize the worldline formalism to manifolds with boundaries. We considered the flat manifold $\mathbb{R}_{+} \times \mathbb{R}^{D-1}$ and made use of an image charge to extend the problem to $\mathbb{R}^{D}$. Smooth potentials on $\mathbb{R}_{+} \times \mathbb{R}^{D-1}$ extend to potentials on $\mathbb{R}^{D}$ which are generically not smooth at $y=0$. Our proposal to deal with this situation is in principle applicable to any perturbative order, but the explicit calculations become increasingly harder as the perturbative order increases. It could be useful to develop simpler strategies to evaluate the path integral in the presence of nonsmooth potentials. This would make it easier to extend the method to curved manifolds with boundaries. Nevertheless we have been able to show the feasibility of the worldline formalism in the presence of boundaries, and used it to calculate two new coefficients, $A_{3}$ and $A_{\frac{7}{2}}$, for a flat space hamiltonian with a generic scalar potential. On top of using curved manifolds, one could try to extend the worldline formalism on spaces with boundaries to include fields with spin and use more generic boundary conditions.

\section{Acknowledgments}

OC would like to thank the organizers of the Simons Workshop in Mathematics and Physics 2006, held at SUNY at Stony Brook, for hospitality and partial support while parts of this work were completed. PAGP acknowledges financial support from CONICET and partial support from UNLP (Subsidio a Jóvenes Investigadores and Proj. 11/X381) and from Coimbra Group. PAGP would also like to thank the Dipartimento di Fisica dell'Università di Bologna for friendly hospitality.

\section{References}

[1] C. Schubert, Perturbative quantum field theory in the string-inspired formalism, Phys. Rept. 355 (2001) 73 [hep-th/0101036]. 
[2] F. Bastianelli and A. Zirotti, Worldline formalism in a gravitational background, Nucl. Phys. B 642 (2002) 372 [hep-th/0205182].

[3] F. Bastianelli, O. Corradini and A. Zirotti, Dimensional regularization for SUSY sigma models and the worldline formalism, Phys. Rev. D 67 (2003) 104009 [hep-th/0211134]; BRST treatment of zero modes for the worldline formalism in curved space, JHEP 01 (2004) 023 [hep-th/0312064].

[4] F. Bastianelli, P. Benincasa and S. Giombi, Worldline approach to vector and antisymmetric tensor fields, JHEP 04 (2005) 010 [hep-th/0503155]; Worldline approach to vector and antisymmetric tensor fields. II, JHEP 10 (2005) 114 [hep-th/0510010].

[5] F. Bastianelli and C. Schubert, One loop photon graviton mixing in an electromagnetic field. I, JHEP 02 (2005) 069 [gr-qc/0412095].

[6] F. Bastianelli, O. Corradini and E. Latini, Higher spin fields from a worldline perspective, hep-th/0701055.

[7] H. Gies, K. Langfeld and L. Moyaerts, Casimir effect on the worldline, JHEP 06 (2003) 018 [hep-th/0303264];

L. Moyaerts, K. Langfeld and H. Gies, Worldline approach to the Casimir effect, hep-th/0311168.

[8] H. Gies and K. Klingmuller, Worldline algorithms for Casimir configurations, Phys. Rev. D 74 (2006) 045002 [quant-ph/0605141].

[9] P. Hor̆ava and E. Witten, Heterotic and type-I string dynamics from eleven dimensions, Nucl. Phys. B 460 (1996) 506 [hep-th/9510209]; Eleven-dimensional supergravity on a manifold with boundary, Nucl. Phys. B 475 (1996) 94 [hep-th/9603142].

[10] N. Arkani-Hamed, A.G. Cohen and H. Georgi, Anomalies on orbifolds, Phys. Lett. B 516 (2001) 395 [hep-th/0103135].

[11] H. Kleinert, Path integrals in quantum mechanics, statistics, polymer physics, and financial markets, World Scientific, Singapore (2004).

[12] M. Chaichian and A. Demichev, Path integrals in physics, Vol. 1: Stochastic processes and quantum mechanics, IOP, Bristol, UK (2001).

[13] H.P. McKean and I.M. Singer, Curvature and the eigenvalues of the Laplacian, J. Diff. Geom. 1 (1967) 43.

[14] G. Kennedy, R. Critchley and J.S. Dowker, Finite temperature field theory with boundaries: stress tensor and surface action renormalization, Ann. Phys. (NY) 125 (1980) 346.

[15] T.P. Branson and P.B. Gilkey, The asymptotics of the Laplacian on a manifold with boundary, Commun. Part. Diff. Equat. 15 (1990) 245.

[16] G. Cognola, L. Vanzo and S. Zerbini, A new algorithm for asymptotic heat kernel expansion for manifolds with boundary, Phys. Lett. B 241 (1990) 381.

[17] D.M. McAvity and H. Osborn, A Dewitt expansion of the heat kernel for manifolds with a boundary, Class. and Quant. Grav. 8 (1991) 603 [Erratum ibid. 9 (1991) 317];

D.M. McAvity, Heat kernel asymptotics for mixed boundary conditions, Class. and Quant. Grav. 9 (1992) 1983.

[18] T.P. Branson, P.B. Gilkey and D.V. Vassilevich, The asymptotics of the laplacian on a manifold with boundary. 2, Boll. Union. Mat. Ital. 11B (1997) 39 [hep-th/9504029]. 
[19] T.P. Branson, P.B. Gilkey, K. Kirsten and D.V. Vassilevich, Heat kernel asymptotics with mixed boundary conditions, Nucl. Phys. B 563 (1999) 603 [hep-th/9906144].

[20] D.V. Vassilevich, Heat kernel expansion: user's manual, Phys. Rept. 388 (2003) 279 [hep-th/0306138].

[21] B.S. DeWitt, in "Relativity, Groups and Topology", Les Houches 1963, ed. B. and C. DeWitt (Gordon Breach, NY, 1964); "Relativity, Groups and Topology II". Les Houches 1983, ed. B. DeWitt and R. Stora (North Holland, Amsterdam, 1984)

[22] D. Fliegner, P. Haberl, M.G. Schmidt and C. Schubert, An improved heat kernel expansion from worldline path integrals, Discourses Math. Appl. 4 (1995) 87 [hep-th/9411177]. 